\title{
QUANTIFICAÇÃO DE AMÔNIA EM INSTALAÇÕES DE PRODUÇÃO DE FRANGOS DE CORTE EM CLIMA QUENTE
}

\author{
Fernanda Campos de Sousa* \\ Ilda de Fátima Ferreira Tinôco** \\ Fátima de Jesus Folgôa Baptista*** \\ Vasco Manuel Fitas da Cruz ${ }^{* * * *}$ \\ Cecília de Fátima Souza Ferreira \\ Alex Lopes da Silva******
}

RESUMO: A qualidade do ar é um dos fatores mais importantes na produção avícola, uma vez que os poluentes aéreos podem alterar as características ideais do ar, favorecendo a susceptibilidade a doenças, além de causar prejuízos na produção. Apesar da grande diversidade de métodos existentes para quantificar a amônia, nem sempre eles podem ser aplicados nas instalações dos países de climas quentes. Assim, ainda são necessários estudos de comparação, adaptação e aplicação de métodos utilizados para quantificar a amônia. Com isso objetivou-se com essa revisão apresentar os métodos mais comumente utilizados para determinar a concentração e a emissão de amônia em instalações de produção animal em todo o mundo e ao mesmo tempo avaliar a sua aplicação nos aviários abertos utilizados nos países de climas quentes. A escolha do método mais adequado deve ser baseada considerando-se os fatores relevantes para a determinação da amônia. Diante da maior dificuldade na determinação da taxa de ventilação nos aviários abertos, as metodologias para avaliação da emissão de amônia que não dependam desse parâmetro são as mais indicadas.

PALAVRAS-CHAVE: Avicultura; Emissão de amônia; Qualidade do ar.

Doutora em Engenharia Agrícola, Docente Adjunta do Departamento de Engenharia Agrícola da Universidade Federal de Viçosa (UFV), Viçosa, MG, Brasil. E-mail: fernanda.sousa@ufv.br.

** Doutora em Ciência Animal, Docente Adjunta do Departamento de Engenharia Agrícola da Universidade Federal de Viçosa (UFV), Viçosa, MG, Brasil.

*** Doutora em Engenharia Rural, Docente Auxiliar do Departamento de Engenharia Rural e Instituto de Ciências Agrárias e Ambientais Mediterrânicas (ICAAM), Universidade de Évora (UEVORA), Évora, Portugal.

${ }^{* * * *}$ Doutor em Engenharia Agrícola, Docente Associado do Departamento de Engenharia Rural e Instituto de Ciências Agrárias e Ambientais Mediterrânicas (ICAAM), Universidade de Evora (UEVORA), Evora, Portugal.

${ }^{* * * * *}$ Doutora em Zootecnia, Docente Associada do Departamento de Engenharia Agrícola da Universidade Federal de Viçosa (UFV), Viçosa, MG, Brasil.

${ }^{* * * * * *}$ Doutor em Zootecnia, Docente Adjunto do Departamento de Produção Animal, Instituto de Zootecnia, Universidade Federal Rural do Rio de Janeiro (UFRRJ), Seropédica, RJ, Brasil. 


\title{
QUANTIFICATION OF AMMONIA IN BROILER PRODUCTION UNITS IN HOT CLIMATE
}

\begin{abstract}
Air quality is one of the most important factors in broiler production. In fact, air pollutants may alter air characteristics, favor susceptibility to diseases and impair production. In spite of the great diversity of methods to quantify ammonia, not all of them may be applied in production units in countries with a hot climate. Studies on comparison, adaptation and application of methods are required to quantify ammonia. Current paper provides the commonest methods used to determine ammonia concentration and emission in animal production installations worldwide and evaluates their application in open aviaries used in countries with a hot climate. Choosing the best method foregrounds relevant factors for the determination of ammonia. In the wake of greater difficulties to determine ventilation rates in open aviaries, methodologies for the evaluation of ammonia emission which do not depend on this parameter are the most indicated.
\end{abstract}

KEY WORDS: Fowl breeding; Ammonia emissions; Air quality.

\section{INTRODUÇÃO}

A qualidade do ar é um dos fatores mais importantes na produção avícola (MENEGALI et al., 2009), uma vez que os poluentes aéreos, eventualmente presentes nos aviários, dependendo de suas concentrações, podem alterar as características ideais do mesmo, favorecendo a susceptibilidade a doenças respiratórias além de causar prejuízos na produção (MIRAGLIOTTA et al., 2004; NÄÄS et al., 2007).

A amônia é um dos poluentes aéreos mais frequentemente encontrados em altas concentrações nos aviários (OWADA et al., 2007). Considerando seu impacto ao ambiente, há mais de uma década as emissões de amônia têm sido foco de estudos de pesquisadores em diversos países da Europa e América do Norte (SCHOLTENS, 2004; MOSQUERA et al., 2005; FAULKNER; SHAW, 2008) onde já se procedeu a realização de inventários sobre emissões de gases, tendo seus protocolos referentes a emissões já estabelecidos.

Para as regiões de clima quente ou tropical, como é o caso do Brasil, existem ainda poucos estudos e métodos para determinação das emissões de amônia 
(BARRETO-MENDES et al., 2014), além de que, os estudos relacionados à qualidade do ar pela produção de carne de frango ainda são limitados a algumas iniciativas relacionadas à saúde dos animais e trabalhadores ou problemas de odores nas proximidades das instalações.

Considerando que o conhecimento dos níveis de emissão constitui um dos fatores norteadores principais para que os órgãos nacionais e internacionais possam exercer o controle ambiental e eliminar barreiras na comercialização de produtos da cadeia avícola (OSORIO-SARAZ et al., 2014), e também para manter a posição de maior exportador de carne de frango, o Brasil deve adequar-se às normas e exigências internacionais atendendo aos padrões de qualidade exigidos, aos requisitos de bem-estar animal e às questões ambientais relacionadas principalmente à qualidade do ar.

Com isso, são necessárias pesquisas nessas áreas, que minimizem os impactos ao meio ambiente, visando qualidade do ambiente de produção e, assim, menor emissão de gases com potencial efeito estufa para o planeta. Buscando sustentabilidade de produção, tanto em termos de segurança alimentar, garantindo um custo alimentar acessível à população em qualquer classe, como também a preservação do meio ambiente para gerações futuras (MARÍN, 2011). Portanto objetivou-se com essa revisão levantar os principais métodos e metodologias para determinação da concentração e emissão de amônia adotados nas instalações de produção animal no mundo e ao mesmo tempo avaliar a sua aplicação nos aviários abertos de produção de frangos de corte utilizados nos países de climas quentes.

\section{REVISÃO BIBLIOGRÁFICA}

\subsection{CONCENTRAÇÃO DE AMÔNIA}

As medições referentes à concentração de amônia no ambiente são dificultadas principalmente pelo equilíbrio que existe entre a amônia e as partículas gasosas presentes no ar que podem ser voláteis em temperatura ambiente (YAMADA et al., 2012). Apesar da grande diversidade de métodos existentes para quantificar a 
concentração de amônia no ambiente a maioria dos métodos ou apresentam custo elevado ou envolvem uma série de etapas em laboratório que podem levar à contaminação da amostra e inviabilizar toda a análise. Além de que, a maioria das metodologias existentes foram desenvolvidas na América do Norte e Europa; são voltadas principalmente às instalações fechadas, utilizadas nessas regiões, onde existe um controle das entradas e saídas de ar na instalação. Assim, ainda são necessários estudos de comparação, adaptação e aplicação de tais metodologias para quantificar a amônia nas instalações abertas, que são predominantemente utilizadas nos países de clima quente (FELIX; CARDOSO, 2004).

\subsubsection{Filter pack}

O método filter pack foi um dos primeiros métodos para quantificação de amônia, com a tentativa de separação da fase gasosa e particulada, porém os dados obtidos com esse método consideram $\mathrm{NH}_{\mathrm{x}}$ total, sem qualquer distinção entre as fases, ou seja, podem quantificar amônio $\left(\mathrm{NH}_{4}\right)$ e amônia $\left(\mathrm{NH}_{3}\right)$ ao mesmo tempo, sem distinção entre eles. São constituídos por filtros impregnados em ácido para reter a amônia. É um método muito utilizado devido ao seu baixo custo e facilidade de preparação e uso. Após a amostragem a concentração de amônia no filtro é determinada por espectrofotômetro (FELIX; CARDOSO, 2004).

\subsubsection{Tubos de difusão passiva}

A técnica de difusão, ou denuders, que são os tubos de difusão passiva, surgiu como uma melhoria do método filter pack por permitir uma amostragem seletiva da fase gasosa e posterior determinação dos particulados por meio da diferenciação do movimento entre gás e partícula. Constituído por um tubo cilíndrico aberto de um dos lados com um meio ácido para reter a amônia por processo de difusão, por isso são considerados métodos passivos (YAMADA et al., 2012). É considerada uma forma barata e simples de monitoramento dos poluentes do ar, inclusive amônia (JIMÉNEZ et al., 2011).

Vários tipos de tubos já foram propostos, o mais utilizado é um tubo em vidro impregnado com solução ácida em seu interior (FELIX; CARDOSO, 2004; YA- 
MADA et al., 2012). Os tubos de difusão apresentam rápida taxa de amostragem, simplicidade de operação e medições mesmo em diferentes direcionamentos do fluxo de ar (YAMADA et al., 2012). Funcionam pelo princípio de difusão molecular onde as moléculas da espécie-alvo difundem-se no gradiente de concentração a partir da concentração do ambiente representada pela extremidade aberta do dispositivo (JIMÉNEZ et al., 2011).

\subsubsection{Amostragem ativa}

A amostragem ativa é considerada como um método convencional para determinar a concentração de amônia, é baseado na amostragem ativa, constituído por um tubo de amostragem, semelhante aos tubos de difusão passiva, porém com grânulos de carbono impregnados em ácido sulfúrico e uma fonte de ventilação própria, a amônia retida é transformada em sulfato de amônio e posteriormente é quantificada por cromatografia iônica. Esse método apresenta vantagens em relação à sua simplicidade de coleta, em curto período de tempo, considerado uma metodologia rápida e ainda pode ser automatizado (OSHA, 2002).

\subsubsection{Sensor ou detector eletroquímico}

Os sensores eletroquímicos são dispositivos que permitem coleta de dados e obtenção de informações com a mínima interferência no sistema estudado, foram designados inicialmente para funcionar por alarme que caracteriza a presença do poluente no ar. Normalmente apresentam a resolução de $1 \mathrm{ppm}$, funcionam baseados na modificação de suas superfícies por diversas camadas ou membranas posicionadas na extremidade do dispositivo que reconhecem a espécie de interesse de maneira seletiva (WHEELER et al., 2003). Apresentam diversas características vantajosas como elevada sensibilidade, custo médio, são portáveis e permitem automação, além de permitir obtenção de informação in situ e em tempo real, são cada vez mais precisos, sensíveis e seletivos (LOWINSOHN; BERTOTTI, 2006).

Apesar da versatilidade dos sensores eletroquímicos, a eficiência de um eletrodo pode ser limitada pelo desgaste gradual da sua superfície, uma consequên- 
cia principalmente da adsorção dos produtos da própria reação de óxido-redução que é utilizada na detecção do poluente (FREIRE et al., 2003). Quando devidamente calibrados e operados, os sensores eletroquímicos apresentam medições confiáveis de concentração de amônia permitindo avaliação instantânea (XIN et al., 2002).

\subsubsection{Monitor fotoacústico}

Os monitores fotoacústicos podem ser utilizados para quantificar a concentração de uma gama de gases (multi-gás) como: dióxido de carbono $\left(\mathrm{CO}_{2}\right)$, óxido nitroso $\left(\mathrm{N}_{2} \mathrm{O}\right)$, metano $\left(\mathrm{CH}_{4}\right)$ e amônia $\left(\mathrm{NH}_{3}\right)$, permitindo a medição sequencial em diferentes pontos da instalação por determinados intervalos de tempo (CALVET et al., 2011). Apresentam custo relativamente elevado, quando comparados a outros métodos comumente adotados para quantificação de amônia (GATES et al., 2005; XIN et al., 2003).

O princípio do funcionamento do monitor fotoacústico é baseado no método de detecção infravermelho fotoacústico, conferindo capacidade de medição de diversos gases que absorvem a radiação infravermelha (AMARAL et al., 2007). Podem ser utilizados para mensurar o monóxido de carbono (CO), quando esse é utilizado como gás marcador para a determinação indireta da taxa de emissão de amônia (SARAZ et al., 2013).

\subsubsection{Cromatografia gasosa}

A técnica de cromatografia gasosa envolve um detector capaz de responder a determinado composto de maneira proporcional a sua concentração no ambiente, realiza análises qualitativas e quantitativas de forma rápida na determinação de compostos presentes em concentrações relativamente baixas em diferentes amostragens, o método direto envolve a preparação de uma série de soluções padrão com concentração próxima ao composto a ser analisado (LIGIERO et al., 2009). Essa técnica é utilizada para determinar tanto a concentração de amônia nos ambientes quanto a concentração de amônia capturada nos coletores, bem como para determinar a concentração de hexafluoreto de enxofre $\left(\mathrm{SF}_{6}\right)$, quando esse é utilizado 
como gás marcador para determinar a taxa de emissão de amônia de forma indireta (SARAZ et al., 2013).

\subsubsection{Técnicas de micro difusão ou armadilha de ácido ou borbulhadores}

Diferentes adaptações da câmara de fluxo são utilizadas em nível de pesquisa para quantificar a amônia da cama nas instalações de produção animal no Brasil. É baseado no método de fixação de amônia por microdifusão e quantificação por titulação ácido-base, onde amostras de cama são incubadas em ambiente fechado junto a substância de fixação de amônia volatizada, podendo-se utilizar ácido bórico como substância de fixação, após a incubação a quantidade de amônia é determinada por titulação (HERNANDES; CAZETTTA, 2001).

Essa técnica apresenta boa precisão e exatidão, é acessível, uma vez que utiliza materiais de baixo custo e reagentes acessíveis, pode ser utilizada em condições de campo desde que a amônia produzida possa ser confinada no ambiente de incubação para que possa ser fixada pelo ácido bórico (HERNANDES; CAZETTA, 2001).

A utilização da técnica de mensuração conhecida como armadilha ácida, constituída por um tubo de ensaio com ácido ortofosfórico ligado a bombas para borbulhamento de ar no ácido, foi considerada a técnica mais adequada para mensurar as perdas de amônia nas instalações de frangos de corte (NICHOLSON et al., 2004).

O Método Saraz ou SMDAE (Saraz method for determination of ammonia emissions) é uma adaptação do método de fluxo passivo baseado no método de difusão de massa e no nitrogênio amoniacal total volatilizado e capturado. $\mathrm{O}$ equipamento é constituído por tubo e esponjas impregnadas em solução de ácido sulfúrico e glicerina para fixação da amônia quantificada por titulação ácido base (OSORIO-SARAZ et al., 2014).

\subsection{EMISSÃO DE AMÔNIA}

Na determinação da emissão de amônia, maior atenção deve ser dada às medições da ventilação nas instalações abertas que é dificultada devido às condições 
instáveis do vento, principalmente nos aviários de frangos de corte nos países de clima quente, pois os galpões são predominantemente abertos e quando fechados adotam a utilização de cortinas, podendo funcionar como instalações híbridas. A necessidade da determinação exata da taxa de ventilação de alguns desses métodos para calcular a emissão de amônia demanda uma identificação exata dos pontos de entrada e saída de ar, sendo que esses praticamente não podem ser definidos com clareza nas instalações abertas para produção de frangos de corte das regiões de clima quente, como é o caso do Brasil (CALVET et al., 2011). Contornando esses problemas referentes à determinação da velocidade do ar, na ausência de outros métodos confiáveis e pela falta de protocolos de referência para tais condições, os métodos já protocolados nos países da América do Norte e Europa podem ser aplicados para determinar a emissão de amônia nas instalações de produção de frangos de corte.

\subsubsection{Produto entre taxa de ventilação e diferença de concentração}

O protocolo usado atualmente na Europa para quantificar as emissões de $\mathrm{NH}_{3}$ provenientes das instalações de produção animal especifica que as medições devem ser realizadas pela taxa de ventilação e pela concentração média de $\mathrm{NH}_{3}$ do ar que entra e sai da instalação. A emissão de $\mathrm{NH}_{3}$ é então calculada como o produto da taxa de ventilação e a concentração média de $\mathrm{NH}_{3}$ (MOSQUERA et al., 2005).

O principal desafio da aplicação desse protocolo nas instalações de frangos de corte com ventilação natural no Brasil é dada à dificuldade na determinação da taxa de ventilação necessária para o cálculo da emissão de amônia. Existem algumas técnicas que podem ser aplicadas para determinar a velocidade do ar em instalações com ventilação natural, entre elas: diferença de pressão, uso de anemômetros de fio quente, ultrassom e métodos de gases marcadores (OGINK et al., 2013):

- O método de diferença de pressão depende das aberturas de entrada e saída de ar para determinar a diferença de pressão entre o interior e o exterior da instalação, porém, com a falta de uniformidade e velocidade do ar nas instalações com ventilação natural, esse método não é confiável para estimar a taxa de ventilação nas instalações abertas de clima quente. 
- A utilização de anemômetro de fio quente pode se tornar onerosa uma vez que é necessário um sensor tridimensional para suprimir as variações na direção do vento, assim uma alternativa mais econômica é o sensor ultrassom por ser mais barato e robusto em relação ao anemômetro tridimensional.

- As técnicas com gás marcador são as mais utilizadas e recomendadas para medir a taxa de ventilação em instalações com ventilação natural. Esse método é baseado no princípio de conservação de massa no processo de ventilação. A taxa de ventilação é determinada em função do monitoramento tanto da taxa de injeção quanto da concentração do gás marcador adicionado ao ambiente. Entre os gases que podem ser utilizados como gases marcadores incluem: óxido nitroso $\left(\mathrm{N}_{2} \mathrm{O}\right)$, monóxido de carbono (CO), dióxido de carbono $\left(\mathrm{CO}_{2}\right)$, hexafluoreto de enxofre $\left(\mathrm{SF}_{6}\right)$, entre outros (MENDES et al., 2015; MOSQUERA et al., 2005; OGINK et al., 2013; SARAZ et al., 2013).

\subsubsection{Gás Marcador}

O método de gás marcador, ou gás traçador, é o mais utilizado para quantificar as emissões de amônia nas instalações com sistema de ventilação natural, é baseado no equilíbrio de massas, onde um gás marcador de origem natural ou artificial é injetado na instalação a uma taxa constante $\left(Q_{\text {tracer }}\right)$ e são retiradas amostras para determinar a concentração do gás marcador $\left(\mathrm{C}_{\text {tracer }}\right)$ e a concentração de amônia $\left(\mathrm{C}_{N H 3}\right)$ conforme Equação 1.

$$
Q_{\mathrm{NH} 3}=\frac{C_{\mathrm{NH}_{3}}}{C_{\text {tracer }}} Q_{\text {tracer }}
$$

Onde:

$\mathrm{Q}_{N H 3}$ - Emissão de amônia $\left(\mathrm{m}^{3} \cdot \mathrm{s}^{-1}\right)$

$\mathrm{C}_{\mathrm{NH} 3}$ - Concentração de amônia (ppm)

$\mathrm{C}_{\text {tracer }}$ - Concentração do gás marcador (ppm)

$\mathrm{Q}_{\text {tracer }}$ - Taxa de injeção do gás marcador $\left(\mathrm{m}^{3} \cdot \mathrm{s}^{-1}\right)$. 
Para que essas amostras sejam representativas o ponto de medição deve ser o mais longe possível das fontes de amônia e do gás marcador a fim de garantir uma homogeneização entre os gases na instalação. Normalmente o gás marcador é injetado no chão próximo aos dejetos e a coleta das amostras é feita no ponto mais alto da instalação, próximo a cumeeira do telhado. Para obter uma concentração média no interior da instalação Mosquera et al. (2005) recomendam utilizar um ponto de coleta a cada $10 \mathrm{~m}^{2}$.

Com medições contínuas da concentração de amônia e da taxa de ventilação, os resultados obtidos com esse método tornam-se precisos e confiáveis. Uma vantagem desse método é que ele possibilita o conhecimento da dinâmica da emissão de amônia (MOSQUERA et al., 2005).

Um cuidado maior deve ser dado ao fato de que a mistura entre os gases pode ocorrer a favor do vento, exigindo maior similaridade nos padrões de origem do marcador e da concentração da amônia que será mensurada. Pelo método da razão de gás marcador não há necessidade de monitoramento da taxa de ventilação para estimar a emissão de amônia, desde que haja uma medição precisa da velocidade de liberação do gás marcador e das concentrações tanto do poluente como da amônia (MENDES et al., 2015). Para as instalações com ventilação natural recomenda-se a utilização da técnica de gás marcador desde que as medições sejam feitas no perímetro da instalação e na direção predominante dos ventos (SARAZ et al., 2013).

A principal vantagem da utilização do método da razão do gás marcador nas instalações de frangos de corte, típicas do Brasil, é que ele estima a emissão do poluente diretamente, sem precisar da taxa de ventilação do ar. Assim, como no método para determinação da taxa de ventilação o ponto mais representativo para as medições das concentrações é na região mais próxima a à saída do ar. O método de marcadores de gás é o mais indicado e mais provável de se tornar um método de referência para as instalações abertas com ventilação natural, porém ainda necessitam de aperfeiçoamento (OGINK et al., 2013; CALVET et al., 2013; MENDES et al., 2015).

\subsubsection{Métodos de balanço de massa}

O método de balanço de massa considera todas as formas de nitrogênio geradas na instalação, para isso são utilizados modelos matemáticos com base em 
transferência de massa para determinar as emissões de amônia (SARAZ et al., 2013). Uma forma simples de balanço de amônia, utilizado nas instalações onde é possível a determinação da taxa de ventilação, demanda medições das concentrações de amônia e da taxa de ventilação do ambiente (CALVET et al., 2011).

A técnica mais aplicada no Brasil baseia-se no balanço de massa de um gás marcador com uma taxa de liberação conhecida, o gás mais comumente utilizado é o $\mathrm{CO}_{2}$ produzido metabolicamente, por apresentar vantagens de homogeneidade com o ar, custos reduzidos, e ainda, por estar prontamente disponível nos confinamentos de animais (BARRETO-MENDES et al., 2014). Os métodos de balanço de massa de $\mathrm{CO}_{2}$ podem ser aplicados nas instalações de frangos de corte com ventilação natural, pois são consideradas as medidas das concentrações de dióxido de carbono em função do animal e dos dejetos como base para a estimativa da taxa de ventilação, conforme Equação 2 (MENDES et al., 2014; PEDERSEN et al., 2008).

$$
Q=\frac{A \cdot\left(\mathrm{CO}_{2}\right)_{\text {metabolic }}\left(\mathrm{CO}_{2}\right)_{\text {litter }}}{\Delta\left[\mathrm{CO}_{2}\right]}
$$

Onde:

$Q$ - Taxa de ventilação da instalação $\left(\mathrm{m}^{3} \mathrm{~d}^{-1} a v \mathrm{e}^{-1}\right)$

$A$ - Atividade relativa do animal; adimensional (Pedersen et al., 2008)

$\left(\mathrm{CO}_{2}\right)_{\text {metabolic }}$ - Produção metabólica de $\mathrm{CO}_{2}\left(\mathrm{~m}^{3} \mathrm{~d}^{-1}\right.$ ave $\left.\mathrm{e}^{-1}\right)$

$\left(\mathrm{CO}_{2}\right)_{\text {litter }}-\mathrm{CO}_{2}$ liberado pelos dejetos $\left(\mathrm{m}^{3} \mathrm{~d}^{-1} \mathrm{ave}^{-1}\right)$

$\Delta\left[\mathrm{CO}_{2}\right]=\left(\left[\mathrm{CO}_{2}\right]_{\text {dentro }}-\left[\mathrm{CO}_{2}\right]_{\text {fora }}\right) \mathrm{CO}_{2}$ no interior e exterior da instalação $\left(\mathrm{ppm}_{\mathrm{v}}\right)$

Assim, a partir da taxa de ventilação determinada, a taxa de emissão de amônia pode ser calculada segundo a Equação 3 .

$$
N H_{3} E R=\frac{Q \cdot \Delta\left[\mathrm{NH}_{3}\right] \cdot W_{\mathrm{NH}_{3}}}{V_{\mathrm{NH}_{3}}}
$$


Onde:

$Q$ - Taxa de ventilação da instalação $\left(\mathrm{m}^{3} \mathrm{~d}^{-1} a v \mathrm{e}^{-1}\right)$

$\mathrm{NH}_{3} E R$ - Taxa de emissão de amônia $\left(\mathrm{g}\right.$ ave $\left.\mathrm{e}^{-1} \mathrm{~d}^{-1}\right)$

$\Delta\left[\mathrm{NH}_{3}\right]$ - Diferença de $\mathrm{NH}_{3}$ na entrada e saída da instalação $\left(\mathrm{ppm}_{\mathrm{v}}\right)$

$W_{N H 3}$ - Peso molecular de $\mathrm{NH}_{3}\left(17.031 \mathrm{~g} \mathrm{~mol}^{-1}\right)$

$V_{N H 3}$ - Volume molar $\mathrm{NH}_{3}$ à temperatura $\left(25^{\circ} \mathrm{C}\right)$ e pressão $\left(1 \mathrm{~atm} \mathrm{e} 0,0245 \mathrm{~m}^{3} \mathrm{~mol}^{-1}\right)$

$\mathrm{O}$ método de balanço de massa de $\mathrm{CO}_{2}$ para determinar as emissões de amônia nas instalações de produção de frangos de corte mostra-se eficiente e pode ser aplicado nas instalações com ventilação natural (MENDES et al., 2014). Entretanto os principais desafios na utilização do $\mathrm{CO}_{2}$ no balanço de massa são relacionados à estimativa correta do $\mathrm{CO}_{2}$ metabólico que depende da atividade relativa do animal e à localização correta do ponto de medição de sua concentração (BARRETO-MENDES et al., 2014).

\subsubsection{Amostradores de fluxo passivo}

Os amostradores de fluxo passivo (PFS) são baseados na difusão de uma superfície de reação, constituídos por tubos de amostragem com material absorvente e solução de fixação de amônia, como tiras de papel filtro embebido em ácido sulfúrico, em resumo, utilizam um tubo revestido com ácido em seu interior (OGINK et al., 2013; MOSQUERA et al., 2005). Segundo o princípio de captação da amônia na mesma velocidade da corrente de ar na instalação, eles não necessitam de medições de taxa de ventilação, o que permite sua aplicação nas instalações com ventilação natural, praticadas no Brasil, onde o vento apresenta características complexas, em termos de direção e velocidade, e com isso é difícil sua determinação. Assim, a taxa de captura de amônia depende somente da concentração de amônia e da velocidade do vento que flui sobre o tubo (OGINK et al., 2013; MOSQUERA et al., 2005), podendo não ocorrer nenhuma evidência de viés de turbulência induzida pelo vento (JIMÉNEZ et al., 2011).

Vários tipos de tubos já foram propostos, porém o mais utilizado trata-se de um tubo em vidro impregnado com solução ácida em seu interior (FELIX; CARDOSO, 2004; YAMADA et al., 2012). O modelo conhecido como Ferm Tube não 
sofre interferência das partículas mas necessita de um longo período de amostragem (FERM, 1979), enquanto o denuder anelar, conhecido como AMANDA (Medição de Amônia por Amostragem Denuder Anelar; WYERS et al., 1993), apresenta menor tempo de amostragem, cerca de 10 a 20 minutos, porém a um custo muito mais elevado que outros (SARAZ et al., 2013). Os mais comumente empregados são filtros de celulose impregnados em solução ácida (FRANCO et al., 2000).

Os amostradores de fluxo passivo são caracterizados como uma alternativa que permite monitorar as emissões de amônia nas instalações de produção animal de forma confiável, simples e a baixo custo (OGINK et al., 2013; JIMÉNEZ et al., 2011; SARAZ et al., 2013). Apresentam diversas vantagens por serem fáceis de construir com materiais simples, fáceis de transportar e manusear além de requererem poucas análises laboratoriais, entretanto como desvantagem desse método está a necessidade de longos períodos de amostragens (MOSQUERA et al., 2005; SARAZ et al., 2013).

\subsubsection{Câmaras de fluxo}

As câmaras de fluxo constituem um método alternativo promissor na determinação das emissões gasosas, especialmente nos sistemas de alojamento onde balanços de massa e técnicas de gases marcadores são inseguras, normalmente apresentam baixo custo de investimento e apresentam facilidade de utilização (OGINK et al., 2013; MOSQUERA et al., 2005; CALVET et al., 2013; PARKER et al., 2013).

Trata-se de uma caixa construída em material inerte, colocada no chão com o ar recirculado no interior da caixa (PARKER et al., 2013). Uma quantidade constante de gás marcador é injetada através de controlador de fluxo de massa. A emissão de amônia é calculada de forma similar ao método de gás marcador, pela razão entre o aumento na concentração de amônia e do gás marcador (MOSQUERA et al., 2005).

O método também é conhecido como caixa de medição rápida, como o próprio nome sugere, permite medições rápidas e confiáveis da emissão de amônia diretamente da fonte (MOSQUERA et al., 2005). Uma das principais limitações na quantificação precisa do método de câmara de fluxo é a necessidade de um grande 
número de medições em diferentes dias e locais, devido à variabilidade espacial no interior da instalação (OGINK et al., 2013).

Para estimar a emissão de amônia em instalações de frangos de corte com ventilação natural utilizando o método de câmaras de fluxo, é necessário estudar as características do fluxo de ar nas regiões próximas ao piso em termos de velocidade e nível de turbulência do ar acima da superfície de emissão. As dimensões da câmara, área superficial e altura, podem afetar o valor estimado da emissão de amônia nas instalações com ventilação natural (MOSQUERA et al., 2005; OGINK et al., 2013).

\subsubsection{Unidade de monitoramento portátil (PMU)}

A unidade de monitoramento portátil (PMU) apresenta custo relativamente baixo quando comparada a outros dispositivos similares (AMARAL et al., 2007; GATES et al., 2005; XIN et al., 2004); tal método é uma boa alternativa para o monitoramento contínuo das emissões de amônia nas instalações de produção animal. A taxa de emissão média é calculada baseada na taxa de ventilação e na concentração de amônia (WHEELER et al., 2003). Pode ser utilizada nas instalações abertas desde que seja considerada a imprecisão na medição da taxa de ventilação, e por isso são mais indicadas para instalações com pressão negativa, onde as laterais dos galpões são fechadas. Quando combinada a determinação da taxa de ventilação de forma correta e confiável, a PMU produz dados de emissão de amônia de qualidade razoável (XIN et al., 2002). Portanto, em instalações fechadas, é uma alternativa confiável que pode ser utilizada no monitoramento contínuo de concentração e emissão de amônia (AMARAL et al., 2007).

Para a avicultura brasileira a PMU pode ser utilizada tanto nas instalações com pressão negativa, onde as laterais são fechadas, quanto nas instalações abertas com ventilação natural, porém, nesse caso é necessário maior número de pontos de amostragem ao longo de toda a instalação, em diferentes alturas e próximo a saídas de ar, associado ao monitoramento constante da velocidade e direção do vento (SARAZ et al., 2013).

\subsubsection{Método Saraz ou SMDAE}


O Método Saraz ou SMDAE (Método Saraz para Determinação das Emissões de Amônia) é uma adaptação do método de fluxo passivo utilizado para determinar o fluxo de amônia nas instalações de produção animal em sistemas de criação que utilizam cama. É baseado no método de difusão de massa e no nitrogênio amoniacal total volatilizado e capturado. O equipamento é constituído por um tubo com esponjas impregnadas em solução de ácido sulfúrico e glicerina. A quantificação da amônia é feita por titulação ácido base e o fluxo de amônia obtido em função da quantidade de amônia capturada no coletor, da área superficial da esponja e do tempo de exposição, o fluxo de amônia é calculado pela Equação 4 (OSORIO-SARAZ et al., 2014).

$$
S M D A E=\frac{N H_{3}}{A \cdot t}
$$

Onde:

SMDAE - Fluxo de amônia $\left(\mathrm{g} \mathrm{NH}_{3} \cdot \mathrm{m}^{-2} \cdot \mathrm{s}^{-1}\right)$

$\mathrm{NH}_{3}$ - Quantidade de amônia capturada $\left(\mathrm{g} \mathrm{NH}_{3}\right)$

A - Área da esponja $\left(\mathrm{m}^{2}\right)$

$\mathrm{t}$ - Tempo de exposição da esponja (s).

O método SMDAE é considerado eficiente e pode ser utilizado como uma alternativa para determinar o fluxo de amônia proveniente de cama de frango em instalações para produção de frangos de corte com ventilação natural (OSORIO-SARAZ et al., 2014), desde que sejam identificadas entradas e saídas de ar para mensuração em no mínimo dois pontos de amostragens. Por causa da possibilidade de maior área de captação, e pela simplicidade do equipamento, essa técnica é menos onerosa que outros métodos (SARAZ et al., 2013).

\section{CONSIDERAÇÕES FINAIS}


Diante da diversidade de métodos existentes para quantificar a amônia, pode-se escolher o mais adequado considerando-se custos, disponibilidade de materiais, período de coleta, finalidade de amostragem, entre outros fatores relevantes para a determinação da amônia.

Contudo, a maior dificuldade nas instalações de produção de frangos de corte nos países de clima quente está na determinação da taxa de ventilação nas instalações abertas por não ser possível um controle eficiente das entradas e saídas de ar.

Por isso, para os aviários abertos, comumente utilizados nos países de climas quentes, as metodologias para avaliação da emissão de amônia que não dependam da determinação da taxa de ventilação são as mais indicadas.

\section{AGRADECIMENTOS}

À Coordenação de Aperfeiçoamento de Pessoal de Nível Superior - Capes, à Fundação de Amparo à Pesquisa do Estado de Minas Gerais - Fapemig e ao Conselho Nacional de Desenvolvimento Científico e Tecnológico - CNPq.

\section{REFERÊNCIAS}

AMARAL, M. F. P.; GATES, R. S.; WILKERSON, E. G.; OVERHULTS, D. G.; TINOCO, I. F. F.; LI, H.; BURNS, R. T.; XIN, H.; EARNEST, J. W. Comparison between two systems for ammonia emission monitoring in broiler houses. In: INTERNATIONAL SYMPOSIUM ON AIR QUALITY AND MANURE MANAGEMENT FOR AGRICULTURE, 2007, Broomfield, Colorado. Anais... Broomfield, Colorado: ASABE: American Society of Agricultural and Biological Engineers, 2007.

BARRETO-MENDES, L.; FERREIRA-TINOCO, I. D. F.; OGINK, N.; OSORIO-HERNANDEZ, R.; OSORIO-SARAZ, J. A. A refined protocol for calculating air flow rate of naturally ventilated broiler barns based on $\mathrm{CO}_{2}$ mass balance. Dyna, Medelliín, v. 81, n. 185, p. 189-195, 2014. 
CALVET, S.; CAMBRA-LÓPEZ, M.; ESTELLÉS, F.; TORRES, A. G. Characterization of gas emissions from a Mediterranean broiler farm. Poult. Sci., New Orleans, v. 90, p. 534-542, 2011.

CALVET, S.; GATES, R. S.; ZHANG, G.; ESTELLÉS, F.; OGINK, N. W. M.; PEDERSEN, S.; BERCKMANS, D. Measuring gas emissions from livestock buildings: A review on uncertainty analysis and error sources. Biosyst. Eng., Springer, v. 116, n. 3, p. 221231, 2013.

FAULKNER, W. B.; SHAW, B. W. Review of ammonia emission factors for United States animal agriculture. Atmos. Environ., Oxford, v. 42, n. 2008, p. 6567-6574, 2008. Doi:10.1016/j.atmosenv.2008.04.021.

FELIX, E. P.; CARDOSO, A. A. Amônia $\left(\mathrm{NH}_{3}\right)$ atmosférica: fontes, transformação, sorvedouros e métodos de análise. Quim. Nova, São Paulo, v. 27, n. 1, p. 123-130, 2004 .

FERM, M. Method for determination of atmospheric ammonia. Atmos. Environ., Oxford, v. 13, n. 10, p. 1385-1393, 1979.

FRANCO, A.; CARDOSO, A. A.; ALLEN, A. G. Extração de amônio de filtros de amostragem, coleta e determinação pelo método da gota suspensa. Eclet. Quim., São Paulo, v. 25, p. 161-170, 2000.

FREIRE, R. S.; PESSOA, C. A.; KUBOTA, L. T. Emprego de monocamadas auto-organizadas no desenvolvimento de sensores eletroquímicos. Quim. Nova, São Paulo, v. 26, n. 3, p. 381-389, 2003.

GATES, R. S.; XIN, H.; CASEY, K. D.; LIANG, Y.; WHEELER, E. F. Method for Measuring Ammonia Emissions from Poultry Houses. Poutry Sci. Assoc., New Orleans, v. 14, n. 4, p. 622-634, 2005.

H. XIN; Y. LIANG; A. TANAKA; R. S. GATES; E. F. WHEELER; K. D. CASEY; A. J. HEBER; J. Q. NI; H. LI. Ammonia emissions from u.s. poultry houses: part i - measurement system and techniques. In: AIR POLLUTION FROM AGRICULTURAL OPERATIONS - 
III, 2003, St. Joseph. Anais... St. Joseph, ASAE: American Society of Agricultural and Biological Engineers, p. 106-115, 2003.

HERNANDES, R.; CAZETTA, J. O. Método simples e acessível para determinar amônia liberada pela cama aviária. Rev. Bras. Zootec., Viçosa, v. 30, n. 3, p. 824-829, 2001.

JIMÉNEZ, A. S.; HEAL, M. R.; BEVERLAND, I. J. Intercomparison study of NOx passive diffusion tubes with chemiluminescence analysers and evaluation of bias factors. Atmos. Environ., Oxford, v. 45, n. 2011, p. 3062-3068, 2011.

LIGIERO, C. B. P.; DOS REIS, L. A.; PARRILHA, G. L.; FILHO, M. B.; CANELA, M. C. Comparação entre métodos de quantificação em cromatografia gasosa: Um experimento para cursos de química. Quim. Nova, São Paulo, v. 32, n. 5, p. 1338-1341, 2009.

LOWINSOHN, D.; BERTOTTI, M. Sensores eletroquímicos: Considerações sobre mecanismos de funcionamento e aplicações no monitoramento de espécies químicas em ambientes microscópicos. Quim. Nova, São Paulo, v. 29, n. 6, p. 1318-1325, 2006.

MARÍN, O. L. Z. Caracterização e avaliação do potencial fertilizante e poluente de distintas camas de frango submetidas a reusos sequenciais na zona da mata do estado de Minas Gerais. 2011. 68f. Dissertação (Mestrado em Engenharia Agrícola) - Universidade Federal de Viçosa, Viçosa, 2011.

MENDES, L. B.; EDOUARD, N.; OGINK, N. W. M.; VAN DOOREN, H. J. C.; TINÔCO, I. D. F. F.; MOSQUERA, J. Spatial variability of mixing ratios of ammonia and tracer gases in a naturally ventilated dairy cow barn. Biosyst. Eng., Springer, v. 129, n. 2015, p. 360-369, 2015.

MENDES, L. B.; TINOCO, I. F. F.; OGINK, N. W. M.; ROCHA, K. S. O.; OSORIO S. J. A.; SANTOS, M. S. Ammonia emissions from a naturally and a mechanically ventilated broiler house in Brazil. Rev. Bras. Eng. Agrícola e Ambient., Campina Grande, v. 18, n. 11, p. 1179-1185, 2014.

MENEGALI, I.; TINÔCO, I. D. F. F.; BAÊTA, F. C.; CECON, P. R.; GUIMARÃES, M. C. D. C.; CORDEIRO, M. B. Ambiente térmico e concentração de gases em instalações 
para frangos de corte no período de aquecimento. Rev. Bras. Eng. Agrícola e Ambient., Campina Grande, v. 13, suplemento, p. 984-990, 2009. Doi:10.1590/S141543662009000700022.

MIRAGLIOTTA, M. Y.; NÄÄS, I. A.; MURAYAMA, M. C.; MOURA, D. J. Software para estimativa de emissão de amônia. Rev. Bras. Agroinformática, v. 6, n. 2, p. 79-89, 2004.

MOSQUERA, J.; MONTENY, G. J.; ERISMAN, J. W. Overview and assessment of techniques to measure ammonia emissions from animal houses: the case of the Netherlands. Environ. Pollut., v. 135, n. 2005, p. 381-8, 2005.

NÄÄS, I. A.; MIRAGLIOTTA, M. Y.; BARACHO, M. S.; MOURA, D. J. Ambiência aérea em alojamento de frangos de corte: poeira e gases. Eng. Agrícola., Jaboticabal, v. 27, n. 2, p. 326-335, mai/ago. 2007.

NICHOLSON, F. A.; CHAMBERS, B. J.; WALKER, A. W. Ammonia emissions from broiler litter and laying hen manure management systems. Biosyst. Eng., Springer, v. 89 , n. 2 , p. $175-185,2004$.

OGINK, N. W. M.; MOSQUERA, J.; CALVET, S.; ZHANG, G. Methods for measuring gas emissions from naturally ventilated livestock buildings: Developments over the last decade and perspectives for improvement. Biosyst. Eng., Springer, v. 116, n. 2013, p. 297-308, 2013.

OSHA. Ammonia in Workplace Atmospheres e Solid Sorbent, Method ID-188. National Institute for Occupational Safety and Health (NIOSH). Centers for Disease Control and Prevention, Atlanta, p. 1-3, 2002.

OSORIO-SARAZ, J. A.; FERREIRA-TINOCO, I. D.; FATIMA; GATES, R. S.; OLIVEIRA-ROCHA, K. S.; COMBATT-CABALLERO, E. M.; CAMPOS-DE-SOUSA, F. Adaptation and validation of a methdology for determing ammonia flux generated by litter in naturally ventilated poultry houses. Dyna, Medellín, v. 81, n. 187, p. 137-143, 2014.

OWADA, A. N.; NÄÄS, I. A.; MOURA, D. J.; BARACHO, M. D. S. Estimativa de bem-estar de frango de corte em função da concentração de amônia e grau de luminosidade 
no galpão de produção. Eng. Agrícola., Jaboticabal, v. 27, n. 3, p. 611-618, 2007. Doi:10.1590/S0100-69162007000400003.

PARKER, D.; HAM, J.; WOODBURY, B.; CAI, L.; SPIEHS, M.; RHOADES, M.; TRABUE, S.; CASEY, K.; TODD, R.; COLE, A. Standardization of flux chamber and wind tunnel flux measurements for quantifying volatile organic compound and ammonia emissions from area sources at animal feeding operations. Atmos. Environ., Oxford, v. 66, n. 2013, p. 72-83, 2013.

PEDERSEN, S.; BLANES-VIDAL, V.; JOERGENSEN, H.; CHWALIBOG, A.; HAEUSSERMANN, A.; HEETKAMP, M. J. W.; AARNINK, A. J. A. "Carbon Dioxide Production in Animal Houses: A literature Review". In: AGRICULTURAL ENGINEERING INTERNATIONAL, 2008. Anais... CIGR Ejournal. Manuscript BC 08 008, v. X. p. 1-19, 2008.

SARAZ, J. A. O.; TINÔCO, I. DE F. F.; GATES, R. S.; PAULA, M. O. DE; MENDES, L. B. Evaluation of different methods for determining ammonia emissions in poultry buildings and their applicability to open facilities. Dyna, Medellín, v. 80, n. 178, p. 51-60, 2013.

SCHOLTENS, R. Measuring ammonia emission rates from livestock buildings and manure stores-part 1: development and validation of external tracer ratio, internal tracer ratio and passive flux sampling methods. Atmos. Environ., Oxford, v. 38, n. 2004, p. 3003-3015, 2004. Doi:10.1016/j.atmosenv.2004.02.030

WHEELER, E. F.; ZAJACZKOWSKI, J. S.; TOPPER, P. A.; GATES, R. S.; XIN, H.; CASEY, K. D.; LIANG, Y. Ammonia Emissions from Broiler Houses in Pennsylvania During Cold Weather. In: INTERNATIONAL SYMPOSIUM ON GASEOUS AND ODOUR EMISSIONS FROM ANIMAL PRODUCTION FACILITIES, 2003, Denmark. Anais... Horsens, Jutland, Denmark: 2003.

XIN, H.; LIANG, Y.; GATES, R. S.; WHEELER, E. F. Ammonia Emission from Iowa Layer Houses. In: MIDWEST POULTRY FEDERATION CONVENTION, 2004, St. Paul. Anais... St. Paul, MN: 2004. 
XIN, H.; WANG, T.; GATES, R. S.; WHEELER, E. F. A Portable System for Continuous Ammonia Measurement in the Field. In: AMERICAN SOCIETY OF AGRICULTURAL AND BIOLOGICAL ENGINEERS, 2002, St. Joseph. Anais... St. Joseph, Michigan: ASAE Annual Meeting, 2002.

YAMADA, T.; UCHIYAMA, S.; INABA, Y.; KUNUGITA, N.; NAKAGOME, H.; SETO, H. A diffusive sampling device for measurement of ammonia in air. Atmos. Environ., Oxford, v. 54, n. 2012, p. 629-633, 2012.

WYERS, G. P.; OTJES, R. P.; SLANINA, J. A continuous-flow denuder for the measurements of ambient concentrations and surface-exchange fluxes of ammonia. Atmos. Environ., Oxford, v. 27, n. A, p. 2085-2090, 1993.

Recebido em: 22/03/2016 Aceito em: 13/07/2017 